\title{
Characteristics of the Changes in the Compressive and Tensile Stress of the Construction Sealant under Cyclic Movement
}

\author{
Kohei Yamashita ${ }^{1}$, Hiroyuki Miyauchi ${ }^{2}$, Akihiko Ito $^{3}$, \\ Tomomi Soeta ${ }^{4}$ and Tohru Nakashima ${ }^{1}$
}

\footnotetext{
${ }^{1}$ Performance Polymers (MS) Solutions Vehicle, KANEKA CORPORATION, 2-3-18, Nakanoshima, Kita-ku Osaka 530-8288, Japan, Kohei.Yamashita@kaneka.co.jp, Tohru.Nakashima@kaneka.co.jp

${ }^{2}$ Department of Building, Materials and Components, Building Research Institute, 1 Tachihara, Tsukuba-shi, Ibaraki, 305-0802, Japan, miyauchi@kenken.go.jp

${ }^{3}$ Production Division, Auto Chemical Industry Co.,LTD., 2044-6 Kamiinayoshi, Kasumigaura-Shi, Ibaraki 315-0056, Japan, akihiko.ito@autochem.co.jp

${ }^{4}$ Technology Development Division, Fujita Corporation, 2025-1, Ono, Atsugi City, Kanagawa 2430125, Japan, soeta@fujita.co.jp
}

\begin{abstract}
A compact fatigue testing machine to quantitatively evaluate the effects of this complex degradation of sealants and the load change of the sealant under dynamic fatigue has been developed. The developed fatigue testing machine is compact with dimensions of (width) $100 \times$ (length) $135 \times$ (height) $110 \mathrm{~mm}$. It can be installed in an outdoor exposure test site or in a chamber. Thus, this machine can be used for performing various compounded accelerated degradation tests. We report the use of this testing machine to examine the effects of this complex degradation of sealants and the load change of the sealant under dynamic fatigue.
\end{abstract}

Keywords: Fatigue Testing Machine, Sealant, Joint, Fatigue Resistance.

\section{Introduction}

The factors contributing to the degradation of the construction sealants used in exterior walls can be broadly classified into weathering (heat, ultraviolet rays, moisture, etc.) and joint movement and they work simultaneously in a complex manner. Especially, for dynamic joints, these factors work simultaneously and in a complex manner, and they continue to take effect from the moment that they are filled in the joint until the end of their life.

Current evaluation tests for the sealants mainly evaluate properties after curing in relation to a single degradation factor. No evaluation method for the effect of movement during curing process or one in which complex degradation can be applied had been established yet.

For this reason, we worked on developing a compact fatigue testing machine for the sealant to quantitatively evaluate the effect of complex degradation and load change in the sealants at the time of dynamic fatigue.

Using the developed compact fatigue testing machine the effects of the joint movement during curing on the physical characteristics and the shape of the sealant and the changes in physical properties of the cured sealants were evaluated. The changes in compression and tensile stress of the sealant when repetitive expansion/contraction fatigue (deformation) was applied to the sealing joints with variable fatigue cycle, displacement, and temperature were also measured and examined. 


\section{Overview of the Fatigue Test}

\subsection{Overview of the Fatigue Testing Machine}

Conventional fatigue test machines are all large devices with low versatility and high price. When developing the current fatigue machine, the following points were paid attention to (a) compact size, lightweight, installable in a chamber; (b) easy mechanism; and (c) the price range is such that concerned parties can use the machine in round-robin fashion.

An overview of the developed testing machine is shown in Figure 1 and Table 1. It comprises of (1) fatigue testing machine unit, (2) load measurement device, and (3) PC for measurement purposes. It is capable of measuring the stress (load) on the sealants simultaneously while applying repetitive expansion/contraction deformation.

The dimensions of the fatigue testing machine are $100 \mathrm{~mm}$ (Width) $\times 135 \mathrm{~mm}$ (Length) $\times$ $110 \mathrm{~mm}$ (Height) $\mathrm{mm}$ and it weighs only $1.3 \mathrm{~kg}$ and can be held by one hand. The device runs on AC $100 \mathrm{~V}$ power source or a DC $12 \mathrm{~V}$ battery, so it can be installed for outdoor exposure test and can also be placed in a chamber

Two types of machines with different fatigue cycles were made. One was a fixed-cycle (one cycle/24 hrs) type, in which the number of rotations was controlled only by the combination of a motor equipped with a decelerator and a worm gear. Another was a variable cycle type, in which the number of rotations could be electronically changed with a stepping motor which was combined with the reduction gear ratio of the worm gear. The main unit is separated into

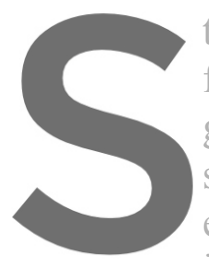
the test unit on the upper fixed platform and that on the low fixed between the mova generated by the recipro section was detected by a load cell. In the co equipped with the decel

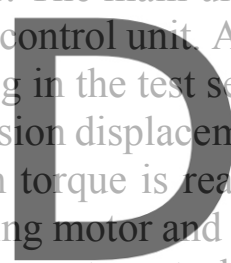
it rotated within the specified range controlled by a displacement control cam attached to a specimen was
, and the load
by the movable
orm gear, and Register fortrion axis The load method is explained as follows, In the displacement mode we used, the displacement was set at $\pm 1.2 \mathrm{~mm}( \pm 10 \%), \pm 2.4 \mathrm{~mm}( \pm 20 \%)$, and $\pm 3.6 \mathrm{~mm}( \pm 30 \%)$ in relation to the joint width of $12 \mathrm{~mm}$. For joint movement cycle, with this machine two levels could be selected, i.e., once per day ( $24 \mathrm{hrs}$ ) and 5 cycles/min (approximately $12 \mathrm{~s}$ ). The latter is the range specified in JIS A 1439.
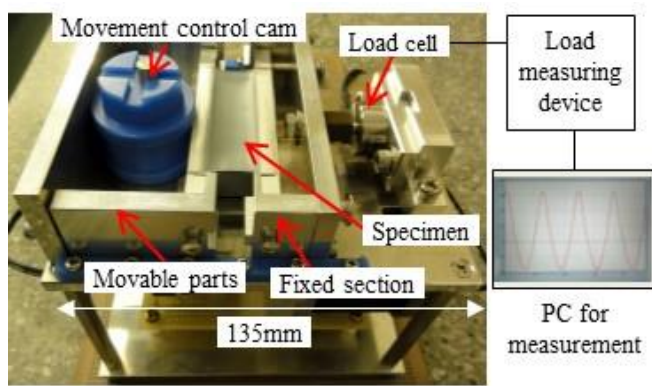

Figure 1. Compact Fatigue Testing Machine (Variable cycle type).
Table 1. Compact Fatigue Testing Machine Specification.

\begin{tabular}{|c|c|c|}
\hline \multirow{2}{*}{$\begin{array}{c}\text { Testing machine } \\
\text { type }\end{array}$} & Fixed Cycle type & Once per day: Decelerator-attachedmotor control \\
\hline & Variable Cycle type & $\begin{array}{l}\text { Once per day to } 5 \text { times/minute: } \\
\text { Stepping motor + electric control }\end{array}$ \\
\hline \multirow{4}{*}{ Load method } & Drive method & Motor+worm gear \\
\hline & Displacement mechanism & Displacement control by cam eccentric motion \\
\hline & Displacement mode & Compressedtensile displacement $\pm 1.2,2.4,3.6 \mathrm{~mm}$ \\
\hline & Cycle & Once per day, 5 times/minute (sine wave) \\
\hline \multirow{2}{*}{ Load } & Load resistance & Maximum $100 \mathrm{~N}$ \\
\hline & Loadmeasurement & Tensile compression load cell (Max.200N) \\
\hline \multirow{2}{*}{ Environment used } & Temperature & -10 to $+50^{\circ} \mathrm{C}$ (depends on motor spec) \\
\hline & Power & AC100V, DC12V battery (forlow load) \\
\hline Others & Dimensions/weight & Width $100 \times$ length $135 \times$ height $110 \mathrm{~mm}, 1.3 \mathrm{~kg}$ \\
\hline
\end{tabular}




\subsection{Sealants Used in the Test}

Silyl-terminated polyether (STPE) type sealants having a silylated polyether main chain with reactive silyl terminal groups as the main ingredient, which forms siloxane bonds through moisture-curing and thereby exhibits rubber elasticity, were selected. It has excellent weatherability, durability, heat resistance, workability, and good paintability on the surface and is widely used for construction sealing materials and elastic adhesives. General structural formula of STPE is shown in Figure 2.

In the test, the two types of sealants were used, i.e., 1-component type (MS-1) and 2component type (MS-2, F-25LM-9030), with tensile properties and relaxation rate shown in Table 2. The tensile properties were measured according to JIS A 1439 (tensile velocity was $5.5 \pm 0.7 \mathrm{~mm} / \mathrm{min}$ ). The stress relaxation rate was obtained from Equation 1. The larger the stress relaxation rate is, more is the reduction in tensile stress generated in the sealant with time.

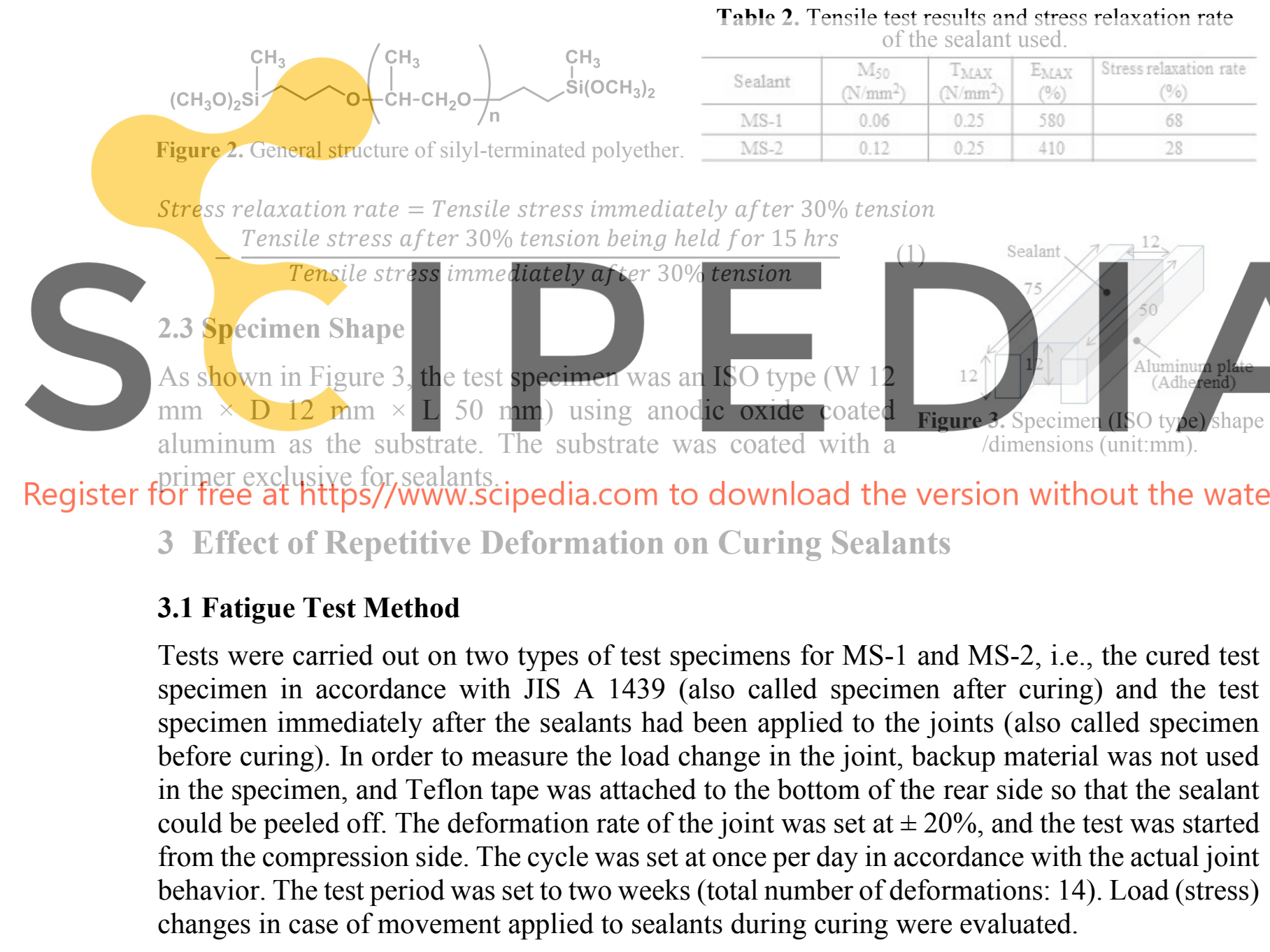

\subsection{Fatigue Test Results}

\subsubsection{Load changes and damage state of the sealant}


Figure 4 shows the load changes in each specimen related to the repetitive deformation. For both MS-1 and MS-2, we can see that the specimen before curing had a reduced load amplitude compared to the specimen after curing, and that it was affected by the movement during the

Table 3. Surface/reverse for specimen before curing after

\begin{tabular}{l|c|c}
\hline & MS-1 Specimen before curing & MS-2 Specimen before curing \\
\hline Surface & & \\
\hline Side & & \\
\hline
\end{tabular}
curing. In particular, the MS-1 specimen before curing showed these tendencies prominently. The conditions of the front and rear face of each pre-cured specimen subjected to fatigue test did not show any change. Meanwhile, the front face of the specimen before curing swelled in a convex shape, as shown in Table 3. The MS-1 specimen before curing had a large hole in the center of the rear side. It was considered that the sealant was damaged due to significant effect of the movement applied during curing. Additionally, the rear face of the MS-2 specimen before curing was deformed to a convex shape.

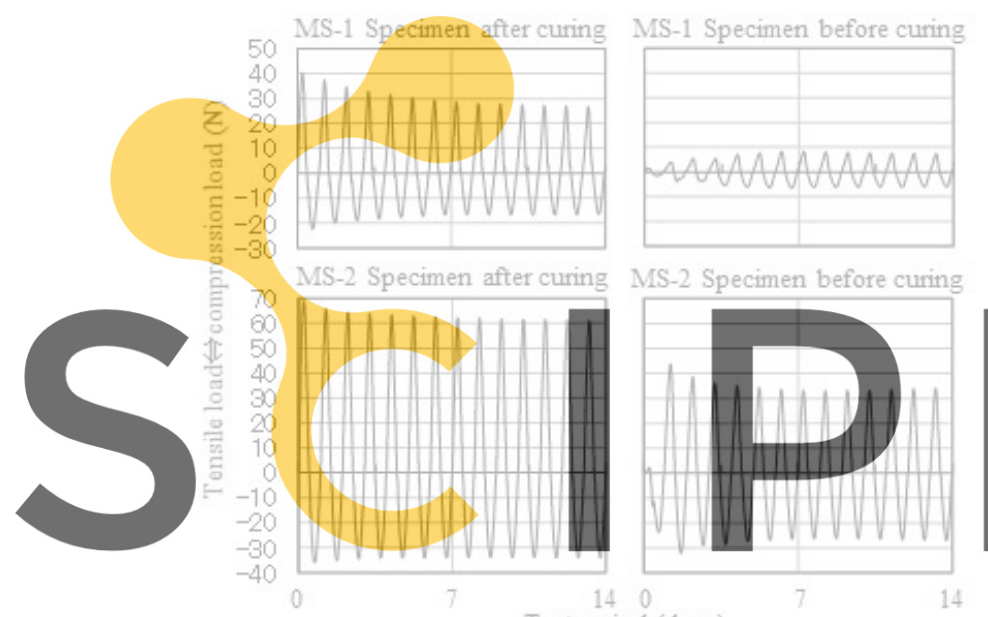

Register for free at https/FWWW.SCIpedia.com to do
Figure 4. Changes in load against repetitive deformation.

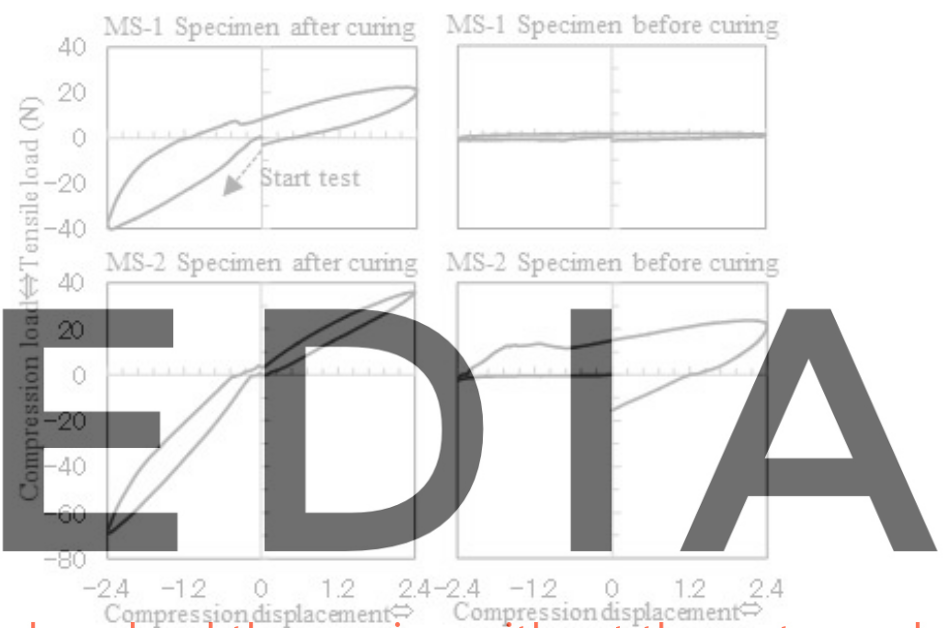

downtoadisthe version withoutethem watermark Figure 5. Displacement and load hysteresis curve for the first repetitive deformation.

\subsubsection{Hysteresis curve of the displacement and load after first fatigue cycle}

Figure 5 shows the hysteresis curve of the displacement and load of the sealants after first repetitive deformation cycle. The initially uncured specimens were slow in expressing the strength compared to the cured specimens, and the load zero value before and after one fatigue cycle did not match. This may be due to change in shape of the sealants or due to damage. Meanwhile, for the cured MS-1 specimen, the difference in the load before and after the tension/compression round-trip was greater compared to that of the cured MS-2 specimen, presumably due to fatigue.

\subsection{Observations Concerning Fatigue Resistance}

\subsubsection{Effect of curing state due to repetitive deformation}

In case of the uncured MS-1 specimen, the sealants hardened gradually from the surface (Figure 6). Since the shape coefficient D/W (D: joint depth, W: joint width) decreases in the initial 
curing period, the sealant was rendered into a state being easily deformed outward, as shown in Figure, once the compression movement was incurred during the curing. Additionally, when the movement occurred during the curing of MS-2, the compression/tensile load ratio changed (Figure 7). This also suggests that the change occurred similarly in the joint cross-section. That is, when it receives the movement during the curing, the subsequent fatigue resistance would be affected.

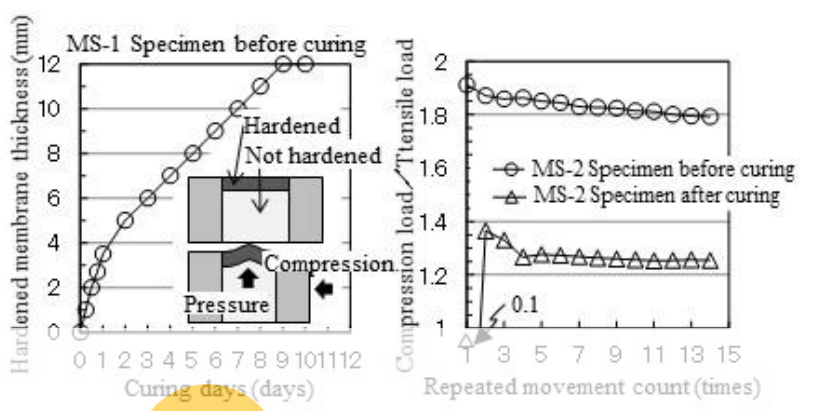

Figure 6. Changes in membrane Figure 7. Changes in compression hickness and joint cross-section change. /tensile load (cycle day 1).

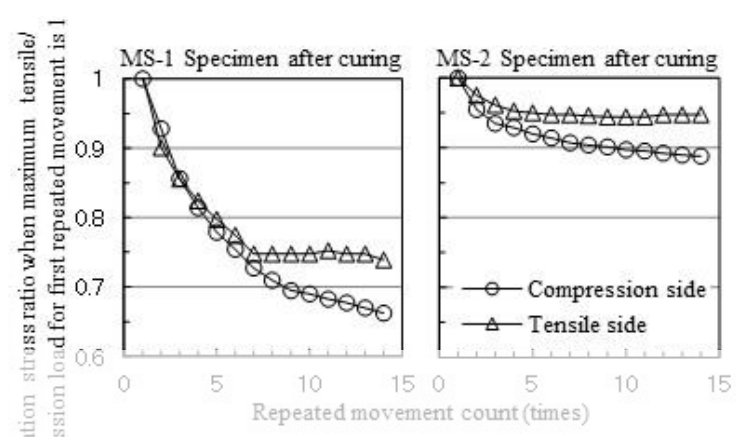

Figure 8. Relationship between relaxation stress ratio and repetitive deformation.

\subsubsection{Effect of stress relaxation due to repetitive deformation}

The relaxation stress ratio (fatigue stress ratio) has been defined as the ratio of the generated stress under the repetitiv expressed as the referen is shown in Figure 8 abo is larger, compared to th decreased after applying repetitiv relaxation ratio on the compression side was gtca aximum stress (load) at the first movement cycle
ship with the number of reptitive deformations
laxation stress ratio of the cured MS-1 specinen
n. Additionally, the relaxation stress rate greatry
. For both specimens, the decrease in the stress
eater thram that on the tensile side, and it can be considered necessary to carry out repetitive fatigue tests to evaluate the effect of stress

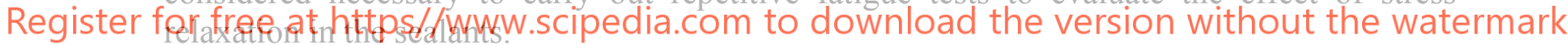

\section{Effect of Repetitive Deformation in Relation to Cured Sealant}

Next, different repetitive deformation were applied to the cured sealant and the compression and tensile stress of the sealant were measured and observed.

\subsection{Fatigue Test Method}

MS-1 and MS-2 described in Section 2.2 were used. The compression and tension deformation rate of the joint was $20 \%$ of joint width, and the fatigue test started from the compression side. The cycle was $12 \mathrm{~s}$, and the maximum number of the fatigue cycles were 10,000 .

\subsubsection{Viscoelasticity Properties of Sealant}

The changes in the stress of the sealant at the first fatigue deformation is shown in Figure 9. For MS-1, maximum compression stress was 2.2 times greater than the maximum tensile stress, and this ratio was 2 times for MS-2. For MS-2, at the displacement zero point (at $6 \mathrm{~s}$ 
where the joint changed from contraction to expansion), the stress became zero, indicating the elasticity. Meanwhile, for MS-1, the changes in the stress preceded the movement, and the tensile stress was generated at the displacement zero point, indicating the tendency to easily undergo compressive setting and the viscoelasticity.

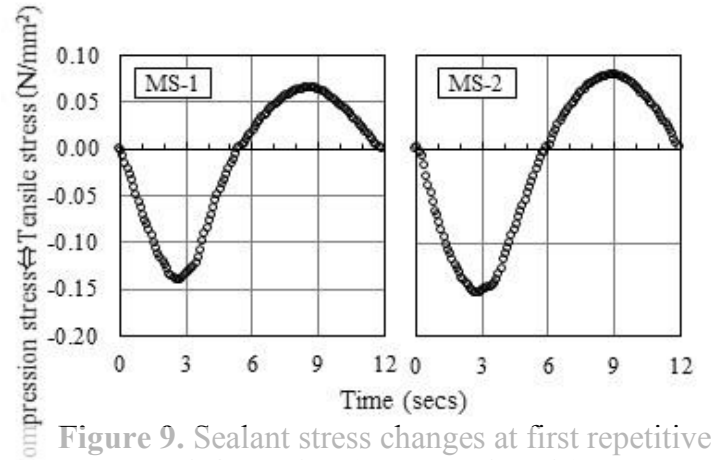

deformation (12-second cycle).
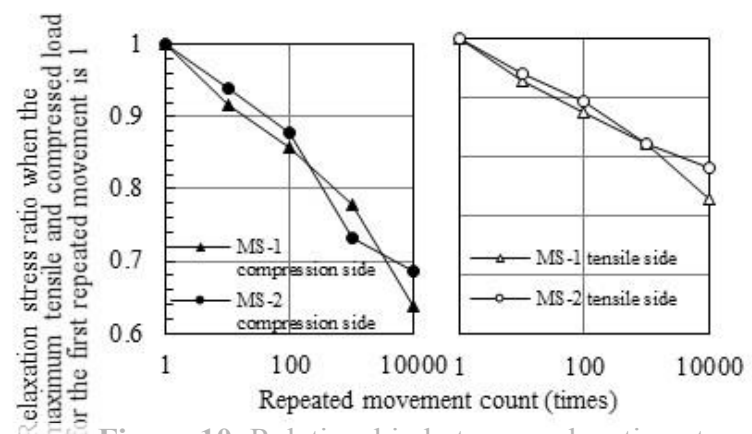

Figure 10. Relationship between relaxation stress ratio and the number of deformation (12-second cycle).

\subsubsection{Sealant Stress Reduction Characteristics in Relation to Repetitive Deformation}

The relationship between the relaxation stress ratio and the number of the repetitive deformation (when maximum tension and compression load for the first repetitive deformation cycle $=1$ ) is shown in Figure 10. For both MS-1 and MS-2, the stress relaxation ratio in the compression side became smaller e

deformations increased. the hysteresis curve of

When the repetitive displacement and stress large in MS-1, compared to MS-2. Additionally,
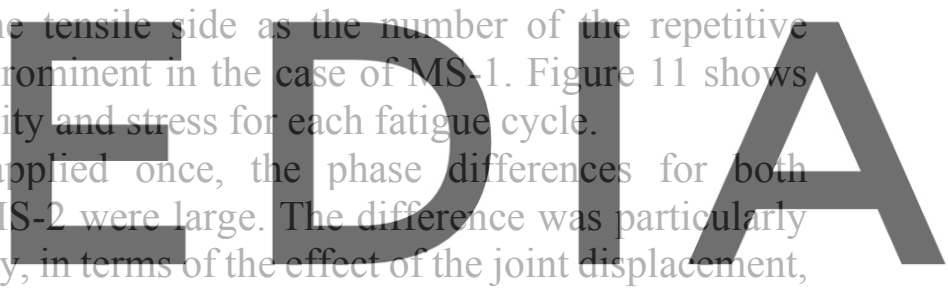

the effect of the compression side is greater than that of the tensile side. Therefore, MS-1 has

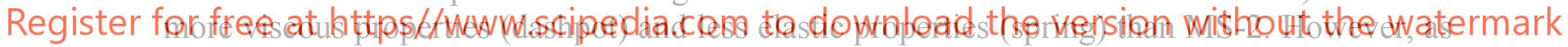
the number of the fatigue cycles increased, phase difference became smaller.

\subsubsection{Effect of the Fatigue Cycle}

Table 4 shows the relationship between the number of test days and total strain energy under test conditions. The 12 -s cycle test had $1 / 10^{\text {th }}$ the duration compared to $1-d$ cycle test, but the total strain energy was five times greater. Figure 12 shows the relaxation stress ratio for the 12$\mathrm{s}$ and 1 day fatigue cycle tests. For MS-2, the stress relaxation ratio at the 14th fatigue cycle in both cases was almost the same. This is because the number of repetitions of the joint deformation may be dominantly influencing the MS-2 stress reduction characteristics, as shown in Figure 10. Meanwhile, for MS-1, the stress relaxation ratio at the 14th fatigue cycle in the 1$\mathrm{d}$ duration cycle test is small, compared to that of the 12-s cycle. This is assumed to be because the fatigue loading time exerts a dominant influence on the MS-1 stress reduction characteristics.

From the above, MS-2 was able to bear the effect of 1 cycle/day fatigue (the actual cycle) based on the test method using a fatigue cycle of 5-6 cycles/min specified in JIS A 1439 under temperature conditions of $23 \pm 2^{\circ} \mathrm{C}$. However, for MS-1, it is may be necessary to execute fatigue tests in accordance with the actual cycle. 


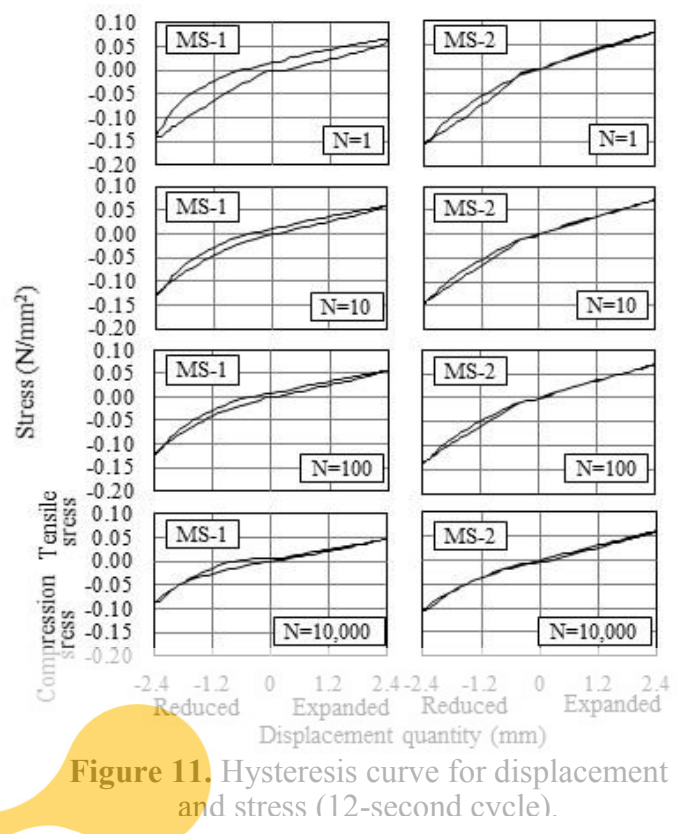

Table 4. Relationship between the number of test days and the strain energy under the load cycles and the maximum deformation cycle in this study.

\begin{tabular}{c|c|c|c}
\hline \multicolumn{2}{c|}{ Test conditions } & \multirow{2}{*}{$\begin{array}{c}\text { Days } \\
\text { (ratio) }\end{array}$} & \multirow{2}{*}{$\begin{array}{c}\text { Total strain } \\
\text { energy }\end{array}$} \\
\hline 12 secs & $10,000(714$ times) & $1.4(1)$ & 5 times \\
\hline 1 day & $14(1)$ & $14(10$ times) & 1 \\
\hline
\end{tabular}

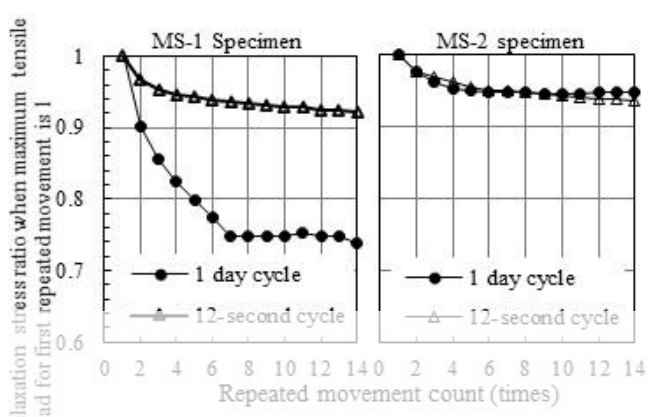

Figure 12. Effect on stress decrease in sealants

\section{Effect of Displacement Quantity, Temperature Conditions and Repetitive} Elongation/Reduction Deformation
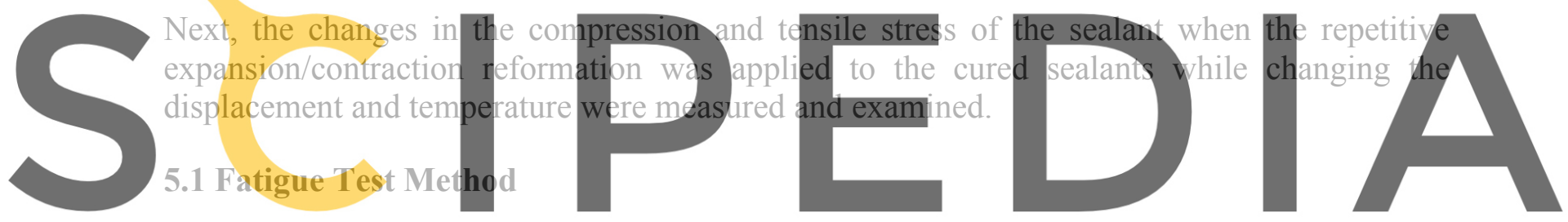

After installing MS-2 specimen in the compact fatigue testing machine, it was kept for sufficient

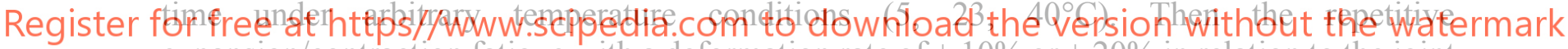
expansion/contraction fatigue with a deformation rate of $\pm 10 \%$ or $\pm 20 \%$ in relation to the joint width was applied from the compression side. The repetition cycle was $12 \mathrm{~s}$, and the maximum number of fatigue cycles were 8,000 to 10,000 (at $23^{\circ} \mathrm{C}, \pm 20 \% 30,000$ times maximum).

\subsection{Fatigue Test Results and Discussion}

\subsubsection{Sealant Stress Reduction Characteristics in Relation to Repetitive deformation}

The relationship between the relaxation stress ratio when the maximum tension stress (load) for the first repetitive deformation cycle was expressed as 1 and the number of the repetitive deformation is shown in Figure 13.

Under the deformation rates of both $\pm 10 \%$ and $\pm 20 \%$, the relaxation stress ratio became lower for each repetition. Also, the deformation rate of \pm $20 \%$ had a smaller relaxation stress rate compared to that of $\pm 10 \%$. Meanwhile, no significant

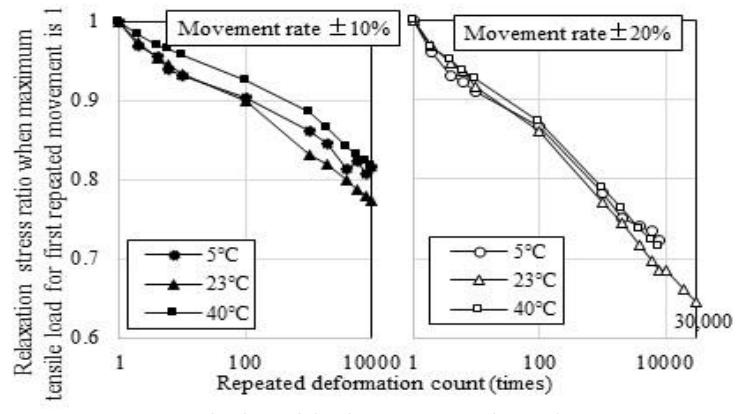

Figure 13. Relationship between relaxation stress rate and the number of deformation (12-second cycle). 
temperature dependency was observed for the relaxation stress ratio.

\subsubsection{Hysteresis Curve of Displacement Quantity and Load}

The hysteresis curves of displacement and the load under various temperature conditions with the deformation rate of $\pm 20 \%$ are shown in Figure 14 .

At $23^{\circ} \mathrm{C}$, the displacement and the phase difference of the load were small up to a fatigue count of 8,000 , whereas the phase difference became large at or above the fatigue count of 20,000. Meanwhile, at $5^{\circ} \mathrm{C}$, the phase difference was large even at the fatigue count of 8,000 , and it was larger than that at $23^{\circ} \mathrm{C}$ and $40^{\circ} \mathrm{C}$.

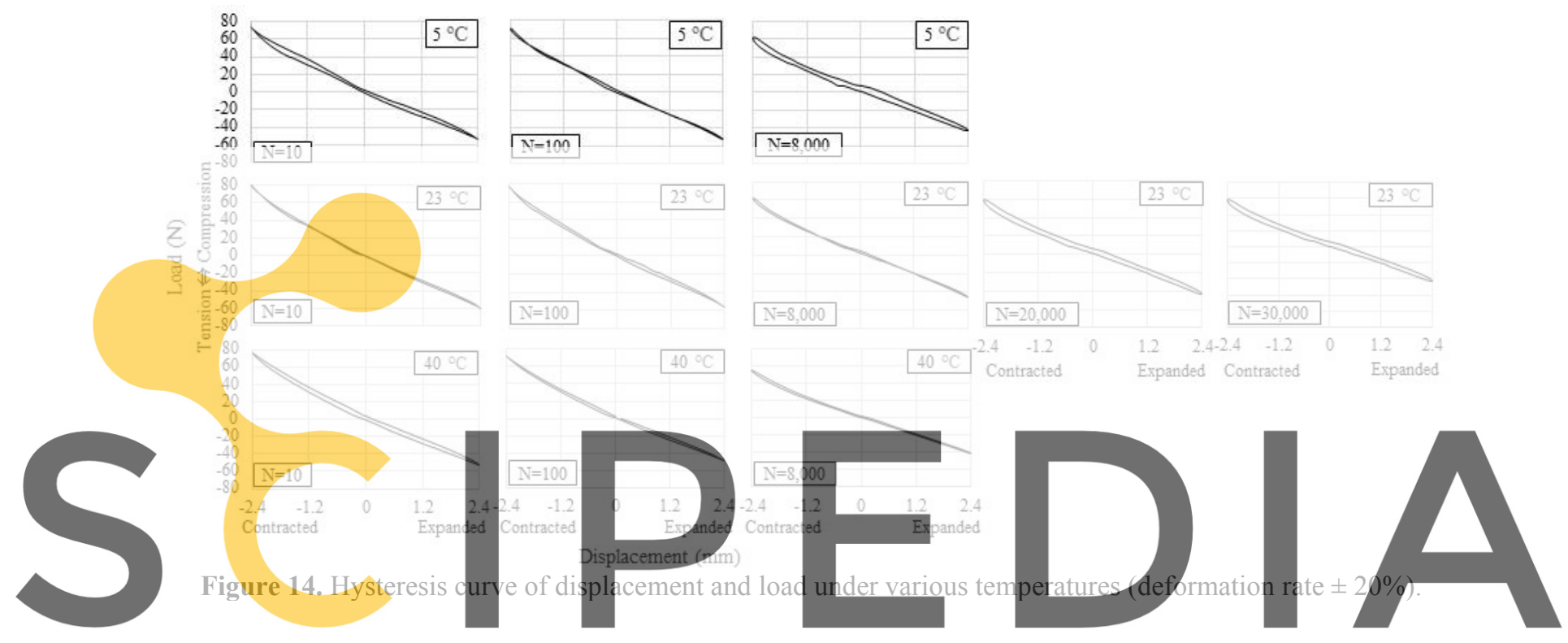

\section{Conclusions}

Register for free at https/fwwwscipedia.com to download the version without the watermark

degradation under outdoor environment. Various methods of utilization can be sought according to the user objectives. For example, the testing machine we developed reproduces the movement of the sealants and can be used in combination with outdoor exposure or in an accelerated deterioration test device. This way It is possible to perform a variety of complex degradation tests and further utilization for future sealing technology can be sought.

\section{ORCID}

Kohei Yamashita: https://orcid.org/0000-0001-9144-4835

\section{References}

Hiroyuki Miyauchi et.al: Measurement of compression-tensile force of sealant when loading cyclic movement to sealed joint by handy-dynamic fatigue testing machine, A-1, Summary of Papers by the Architectural Institute of Japan, pp.1343-1344, 2016

Hitoshi Yamada et.al: Characteristic of compressive-tensile stress change when loading joint movement to sealed joint, Part 1, A-1, Summary of Papers by the Architectural Institute of Japan, pp.903-904, 2017

Kohei Yamashita et.al: Characteristics of compressive and tensile stress change of construction sealant under cyclic movement, Part2, A-1,Summary of Papers by the Architectural Institute of Japan, pp.1033-1034, 2019 